\section{Theory and practice of molecular cytogenetics applied to medicine}

\author{
Molecular Cytogenetics: Protocols and \\ Applications \\ Yao-Shan Fan \\ Humana Press Inc., Totowa, NJ; 2002. 411 pp. \\ \$135.00, hardback. ISBN 1-58829-006-9.
}

Heredity (2003) 91, 437. doi:10.1038/sj.hdy.6800322

\section{Reviewed by E Volpi}

'Molecular Cytogenetics: Protocols and Applications' is an impressive review of molecular cytogenetic techniques and their applications for both clinical research and diagnostic and prognostic purposes.

The book is multiauthored and divided into four sections. Part 1 (Basic Concepts and Techniques) starts with an introductory chapter by the Editor - Yao-Shan Fan - in which he gives a general overview on how, in the past two decades, the introduction of the Fluorescence in situ Hybridisation (FISH) technique and, in particular, its diversification have allowed Cytogenetics to reinvent itself as an important component in many areas of medical practice. The following three chapters are purely technical, with the first two (Chapters 2 and 3 ) describing in overwhelming detail the general principles of probe labelling and different labelling methods, and the third one (Chapter 4 ) covering the general aspects of sample preparation and the hybridisation procedure. These three chapters are a prologue to the comprehensive collection of more specific protocols, which follows in Part 2 (Evolving Techniques and Applications). Included in this second part of the book are 10 chapters, each describing a different technique. The way in which each chapter is structured is very clever (and bound to be very popular with the readers), with the main focus on a step-by-step protocol, preceded by a suitable amount of background information including an overview of the possible applications, and followed by a list of troubleshooting notes (obviously based on the author's firsthand experience and the best I have ever encountered in such a handbook).

All multiauthored books do not have an even quality of form and content, and this work is no exception. For example, although the chapters are generally well written and the protocols clear, I noticed that some of them - in particular, chapter 7 on Spectral Karyotyping and chapter 12 on Multi-Telomere FISH - had more and better explanatory detail and illustrations than others. Readers interested in the Primed In Situ Labeling technique (Chapter 6) would have surely benefited from an enclosed list of suitable primers and Chapter 10 on
Color Banding would have undoubtedly gained in clarity with the addition of an explanatory diagram or a few pictures.

While the first two sections of the book emphasise the methodological aspects of the different molecular cytogenetic techniques, Part 3 (Special Applications in Chromosomal Disorders) and Part 4 (Special Applications in Oncology), although still including detailed protocols, concentrate more on the applications and biological questions they can help answer. Both parts are clear, informative and modern. In addition to wellknown FISH applications in Medical Genetics, for example, Prenatal Diagnosis and Microdeletion Syndromes, Part 3 of the book covers the relatively new area of Molecular Cytogenetics applied to Reproductive Medicine, including as well a chapter on Preimplantation FISH. Part 4, beside more classical topics like interphase FISH studies of CML and CGH for cancer investigations, includes description of experimental strategies to combine molecular cytogenetic essays with histological (Chromogenic In Situ Hybridization) and cytological analysis (Simultaneous Immunophenotyping and FISH).

By gathering such an impressive list of contributions, this book certainly achieves the goal of providing an upto-date collection of protocols and a comprehensive reference source for clinicians and scientists interested in Molecular Cytogenetics in all its guises. Paradoxically, the strength of the book, which is its extreme comprehensiveness, in my respectful opinion, is also its weakness. The pursuit of the broadest possible coverage of this field has inevitably generated some redundancy and has meant that the book's original focus on clinical research and disease management has been compromised. For instance, some of the techniques and protocols described in the first two parts of the book, although interesting, are not strictly pertinent to medical applications, as confirmed by the fact that they are never or very rarely mentioned in Parts 3 and 4 .

Although perhaps a minor criticism, it is perhaps open to argument as to whether etymologically it is truly correct to include within the compass of Molecular Cytogenetics techniques, like Genotyping and Microarrays, which are purely molecular and do not imply any kind of cytological approach. Apart from minor disagreements about the Editor's criteria for the selection of certain items, I enjoyed reading this book very much. I will certainly use it and recommend it to my colleagues. If it has not already become an indispensable resource for clinicians and scientists involved in Molecular Cytogenetics, it soon will.

E Volpi

Molecular Cytogenetics and Microscopy Core, Wellcome Trust, Centre for Human Genetics, University of Oxford, UK 